\title{
PRIME ELEMENTS AND PRIME SEQUENCES IN POLYNOMIAL RINGS
}

\author{
EDWARD D. DAVIS ${ }^{1}$
}

\begin{abstract}
The central question of this note concerns the existence of prime elements in polynomial rings. In it are established for polynomial rings over arbitrary noetherian rings-insofar as is generally possible-certain results concerning bases for maximal ideals, well known for polynomial rings over fields and principal ideal domains. These results may be interpreted geometrically as theorems about normal (and especially smooth) closed points on ruled schemes.
\end{abstract}

Introduction. A complete intersection prime-a prime ideal $P$, generated by $\mathrm{ht}(P)$ elements-can be generated by a regular sequence which remains regular under all permutations. (Rings here are commutative and noetherian.) The (Strong) Prime Sequence Question: Can complete intersection primes be generated by (permutable) prime sequences? (A regular sequence is said to be prime if its initial segments generate prime ideals.) Both versions of the question are presently open; moreover, there is no known instance of the weaker form alone having been decided. The answers are affirmative in several familiar contexts: for ideals contained in the Jacobson radical-such complete intersection primes occur only in integral domains; for maximal ideals in polynomial rings over fields or principal ideal domains; in the coordinate ring of any affine algebraic variety over an infinite ground field. So counterexamples may be difficult to find, especially in domains. (At least for the weaker question, if there is no height 2 counterexample, then there is none at all.)

The present note results from an examination of what seemed to be a potential source of counterexamples: maximal ideals in polynomial rings. It considers maximal ideals $M$, in polynomial rings $A$, over arbitrary noetherian rings, proving: in all instances where general theory guarantees the equivalence of " $A_{M}$ is regular" with " $M$ is a complete intersection", $M$ is generated by a permutable prime sequence. Now finding prime elements, let alone prime sequences, is a difficult, and sometimes impossible, task, even in normal domains. But we shall see that in the cases just mentioned, if $A_{M}$ is normal, then $M$ contains many prime elements, and even has a minimal basis

Received by the editors March 23, 1977 and, in revised form, July 18, 1977.

AMS (MOS) subject classifications (1970). Primary 13B25, 13E05, 14M10.

Key words and phrases. Polynomial ring, maximal ideal, prime element, prime sequence, complete intersection, Gauss' Lemma, ruled affine scheme, normal point, smooth point.

${ }^{1}$ The author gratefully acknowledges the hospitality of the mathematics departments of Louisiana State University and The University of Chicago. 
consisting of such. This last point, which has its geometric interpretation in terms of "ruled" schemes, is really the principal result of this note; the prime sequence theorem emerges, in effect, as a corollary of the proof.

Notation. A regular sequence $\left(x_{1}, \ldots, x_{n}\right)$ is a prime sequence provided that $\left\langle x_{1}, \ldots, x_{i}\right\rangle$ is a prime ideal $(1 \leqslant i \leqslant n)$. (N.B. The ideal generated by the set $\left\{x_{1}, \ldots, x_{n}\right\}$, or by the sequence $\left(x_{1}, \ldots, x_{n}\right)$, is denoted by $\left\langle x_{1}, \ldots, x_{n}\right\rangle$.) A prime element is a regular element which generates a prime ideal. A minimal basis for an ideal is a basis of minimal cardinality.

(1) THEOREM. Let $M$ be a maximal ideal of $A=R[T], R$ a noetherian ring, $T$ an indeterminate, and $P=M \cap R$. Assume: (i) $A_{M}$ (equivalently, $R_{P}$ ) is a domain; (ii) $P$ is maximal; (iii) $A_{M}$ (equivalently, $R_{P}$ ) is normal, or $A / M=$ $R / P$. Then:

(a) $M$ contains infinitely many principal prime ideals (except for the necessarily exceptional case where $\mathrm{ht}(M)=1$ ).

(b) $M$ has a minimal basis consisting of prime elements.

(c) For $A_{M}$ (equivalently, $R_{P}$ ) regular, the basis in (b) can be chosen so that its nonempty subsets generate prime ideals.

For $R$ an affine domain over an infinite field, (1) is a consequence of the following much stronger theorem of Bertini type, which yields affirmative answers to the prime sequence questions for such domains. Recall that the grade (or depth) of an ideal is the length of the longest regular sequence in the ideal. (See [K] for "grade".)

(2) THEOREM. Let I be an ideal in an affine domain over an infinite field. Then I has a minimal basis with the property that each of its subsets having cardinality less than grade $(I)$ generates a prime ideal.

(2) implies that $I$ has a minimal basis containing a regular sequence of length grade $(I)$. This much is true in arbitrary noetherian rings. Armed with this elementary fact, one can produce an inductive proof of (2) by using the Bertini theorem trick-attributed by Swan to Murthy-contained in the proof of [Sn, 1.5]. One should, however, use Seidenberg's Bertini theorem [Sg, Theorem 12] in place of [Sn, 1.1]. (2) is recorded here for expository purposes only; the details of the proof, which shed no light on the more elementary proof of (1), are therefore omitted. It is expected that a future note will treat extensions of (2) to a wider class of rings.

REMARKS ON THE HYPOTHESIS OF (1). (i) is essential: a local ring which contains a prime element must be a domain (Nakayama's Lemma). (ii) and (iii) are automatically satisfied if $R$ is an affine algebra over an algebraically closed field. (ii) is satisfied if $R$ is a Hilbert ring. (See [K] for "Hilbert ring".) (ii) implies [DG]: cardinality of a minimal basis for $M=$ embedding dimension of $A_{M}=1+$ embedding dimension of $R_{P}$. ("Embedding dimension" = "dimension of Zariski tangent space".) (ii) may be assumed 
satisfied if $R$ is a polynomial ring [DG]. If (ii) is not satisfied, then even if $A_{M}$ is regular, $M$ may contain no prime element and may fail to be a complete intersection [E]. The normality part of (iii) may be weakened to: the normalization of $R_{P}$ has a maximal ideal with residue field $R / P$. (See remark following proof of (6).)

Before the proof of (1), three easy corollaries. Given the above remarks, the first is merely a restatement of a special case of (1) in geometric language.

(3) Corollary. Let $X$ be a ruled affine scheme over $Y=\operatorname{Spec}(R)-i . e$, $X=\operatorname{Spec}(R[T])-R$ a noetherian Hilbert ring. Let $x$ be a closed point of $X$ at which $X$ is locally integral, and let $x \mapsto y$, where $X \rightarrow Y$ is the projection. Suppose either that $x$ is a $k(y)$-rational point, or that the normalization of $\operatorname{Spec}\left(\vartheta_{Y_{y}}\right)$ has a $k(y)$-rational closed point. Then $x$ is the intersection of a set of $n$ integral hypersurfaces on $X$, where $n$ is the dimension of the Zariski tangent space of $X$ at $x$. Moreover, if $X$ is smooth at $x$, then this set of hypersurfaces can be chosen so that its nonempty subsets cut out integral subschemes of $X$ which are smooth at $x$.

(4) COROllary. Let $M$ be a maximal ideal of a polynomial ring in $n \geqslant 1$ variables over a regular ring $R$. Then in each of the following cases, $M$ is generated by a permutable prime sequence: (a) $R$ is a Hilbert ring; (b) $n>1$; (c) $\operatorname{dim}(R) \leqslant 1$.

(5) COROllary. Let $R$ be a 2-dimensional, factorial, noetherian domain. Then the strong prime sequence question has an affirmative answer for $R[T]$. (Moreover, if $R$ is regular, then every maximal ideal of $R[T]$ is a complete intersection.)

REMARKS. (4c) is in effect due to Hilbert-Zariski-Endo; for the "classical" construction of $\mathrm{ht}(M)$ generators for $M$ when $R$ is a field [Z] or a principal ideal domain [E], in fact produces a permutable prime sequence. (Exercise: Prove this.) (5) is stated for the sake of recording yet another class of rings for which the prime sequence questions are decided. The parenthesized statement in (5) is true even if $R$ is not factorial [BDG]; but in the nonfactorial case, the prime sequence questions remain open. Given (1) and the remarks on its hypothesis, the proofs of (4) and (5) require little comment. Since a regular ring is a direct sum of domains, for (4) there is no loss in assuming $R$ a domain. Then the only case not covered by (1) is (4c) when $n=\operatorname{dim}(R)=$ $\operatorname{ht}(M)=1$, whence $M \cap R=0$. But then $R$ is semilocal [AT], and hence factorial; whence $M$ is principal. As for (5), there is no problem with primes of height $\neq 2$, even if $R$ is not factorial. If $Q=\langle x, y\rangle$ is a height 2 prime, then $Q=\left\langle x^{\prime}, y^{\prime}\right\rangle$, for some prime factors $x^{\prime}$ of $x$ and $y^{\prime}$ of $y$. Clearly both $\left(x^{\prime}, y^{\prime}\right)$ and $\left(y^{\prime}, x^{\prime}\right)$ are prime sequences. Of course this last remark applies to any height 2 complete intersection prime in a factorial domain.

The proof of (1) requires the following variant of Gauss' Lemma. 
(6) LEMMA. With the hypothesis of (1), assume further that $R$ is a domain. Let $f \in M$ have leading coefficient $a$ unit and generate $M \bmod P A$. Then $f$ is $a$ prime element.

Proof. Claim (i): It suffices to prove that $f$ is irreducible in $K[T], K$ the quotient field of $R$. To see this consider $Q \in \operatorname{Ass}(A /\langle f\rangle)$. Since $Q \in$ $\operatorname{Ass}(A /\langle x\rangle)$ for any $0 \neq x \in Q$, if $0 \neq x \in Q \cap R$, then $Q$ is the extension of some member of $\operatorname{Ass}(R /\langle x\rangle)$. This is impossible because $f$ lies in no extended ideal. So $Q \cap R=0$ for every member of $\operatorname{Ass}(A /\langle f\rangle)$. Therefore all primary components of $\langle f\rangle$ survive in passing from $A$ to $K[T]$. Hence if $f$ is irreducible in $K[T]$, then $\langle f\rangle K[T]$ is a prime ideal, whence so is $\langle f\rangle$. Q.E.D. for Claim 1.

Claim (ii). $f$ is irreducible in $K[T]$. This is clear if $A / M=R / P$; for then $\operatorname{deg}(f)=1$. So assume that $R_{P}$ is normal, in which case, by Gauss' Lemma, it suffices to show that $f$ is irreducible in $R_{P}[T]$. There is no loss in assuming $R=R_{P}$. Let $a \mapsto \bar{a}$ denote the canonical $R$-algebra homomorphism $A \rightarrow$ $(R / P)[T]$. Suppose $f=u v(u, v \in A)$. Then since $\bar{f}=\bar{u} \bar{v}$ is irreducible, it may be assumed that $\bar{u}$ is a unit. Now since $\operatorname{deg}(\bar{f})=\operatorname{deg}(f)$, it follows that $\operatorname{deg}(\bar{u})=\operatorname{deg}(u)$, and so that $u$ is a unit. Q.E.D. for Claim (ii), and hence for (6).

REMARKS. The proof of Claim (i) requires much less than the full hypothesis of (6): it is enough to know that the ideal generated by the coefficients of $f$ is not of grade 1. As for Claim (ii), it suffices to assume: $\bar{f}$ is irreducible; $\operatorname{deg}(\bar{f})=\operatorname{deg}(f)$; there is a maximal ideal $Q$, in the normalization $N$, of $\boldsymbol{R}_{\boldsymbol{P}}$ such that $N / Q=R / P$. (Just substitute $N_{Q}$ for $R_{P}$ in the proof of Claim (ii).) Thus (6) remains valid with the normality hypothesis so weakened. Now the normality hypothesis of (1) enters into its proof only through the use of (6). Hence (1) too remains valid with the same weakening of the normality hypothesis.

Proof of (1). Fix a monic polynomial $g$ that generates $M \bmod P A$. Choose $s \in R-P$ to lie in every member of $\operatorname{Ass}(R)$ except for the unique member $Q$ contained in $P$. Then $R_{s}$ is a domain and $Q R_{s}=0$. Choose $x \in P$ so that $\langle x\rangle+\langle s\rangle=R$. Let $f=s g+x$.

Claim (i). For $y, z \in R,\langle f+s y\rangle=\langle f+s z\rangle \Rightarrow y-z \in Q$.

Proof. This is clear if $R$ is a domain; whence $y-z$ becomes 0 in $R_{s}$. So $y-z \in Q$.

Claim (ii). For $y \in P, f+s y$ is a prime element.

Proof. $f+s y$ is regular since its coefficients generate the unit ideal. Notice that $\langle f+s y\rangle+\langle s\rangle=A$. Hence:

$$
A /\langle f+s y\rangle=A_{s} /\langle f+s y\rangle A_{s} \approx B[T] /\langle h\rangle,
$$

$B=R_{s}, h$ the (canonical) image of $f+s y$ in $B[T]$. By (6)-applied with the roles of $R, P, M, f$ being taken by $B, P B, M B[T], h-h$ is a prime element. So $\langle f+s y\rangle$ is a prime ideal. 
Claims (i) and (ii) show that there are at least as many principal prime ideals in $M$ as there are elements in $P / Q$. This establishes (a). The rest of the proof consists of further applications of the ideas in the proof of Claim (ii).

Rule. Given $s, x, f$ as above, and a sequence $\left(x_{1}, \ldots, x_{n}\right)$ in $P$, define a sequence $\left(f_{1}, \ldots, f_{n}\right)$ in $M$ by setting $f_{i}=f+s x_{i}$.

Now fix such a sequence in $P$ such that $\left\{x_{1}, \ldots, x_{n}\right\}$ is a minimal basis for $P R_{P}$. In the sequel, $s$ and $x$ are always assumed to have the properties prescribed above; (b) and (c) will be established by further restricting $s$ so that the polynomials given by the above rule produce the desired bases for $M$.

To prove (b) further restrict $s$ so that $\left\langle x_{1}, \ldots, x_{n}\right\rangle R_{s}=P R_{s}$. It suffices to show that $M=\left\langle f, f_{1}, \ldots, f_{n}\right\rangle$; for these polynomials are prime elements by Claim (ii), and $n+1$ is the cardinality of a minimal basis for $M$ by [DG]. Now:

$$
M /\left\langle f, f_{1}, \ldots, f_{n}\right\rangle=M A_{s} /\left\langle x_{1}, \ldots, x_{n}, f\right\rangle A_{s}=M A_{s} /\langle P, f\rangle A_{s}=0 .
$$

Q.E.D. for (b).

Suppose now that $R_{P}$ is regular. Then both $\left\{x_{1}, \ldots, x_{n}\right\}$ and $\left\{x_{2}-\right.$ $\left.x_{1}, \ldots, x_{n}-x_{1}, x_{1}\right\}$ are regular systems of parameters for $R_{P}$. Denote generically by $I$ ideals of the form $\left\langle x_{1}, \ldots, x_{j}\right\rangle$ or $\left\langle x_{2}-x_{1}, \ldots, x_{j}-x_{1}\right\rangle$ $(1 \leqslant j \leqslant n)$. Since $I R_{P}$ is a prime ideal, exactly one member of $\operatorname{Ass}(R / I)$ is contained in $P$. Further restrict $s$ so that for each such $I, s$ lies in every member of $\operatorname{Ass}(R / I)$ except for the one contained in $P$. Then $I R_{s}$ will be a prime ideal.

Claim (iii). $\left\langle f_{1}, \ldots, f_{j}, f\right\rangle$ and $\left\langle f_{1}, \ldots, f_{j}\right\rangle$ are prime ideals $(1 \leqslant j \leqslant n)$.

Proof.

$$
\begin{aligned}
A /\left\langle f_{1}, \ldots, f_{j}, f\right\rangle & =A_{s} /\left\langle x_{1}, \ldots, x_{j}, f\right\rangle A_{s} \approx B[T] /\langle h\rangle, \\
B & =R_{s} /\left\langle x_{1}, \ldots, x_{j}\right\rangle R_{s},
\end{aligned}
$$

$h$ the image of $f$ in $B[T]$; and $h$ is a prime element by (6).

$$
\begin{gathered}
A /\left\langle f_{1}, \ldots, f_{j}\right\rangle=A_{s} /\left\langle x_{2}-x_{1}, \ldots, x_{j}-x_{1}, f_{1}\right\rangle A_{s} \approx B[T] /\langle h\rangle, \\
B=R_{s} /\left\langle x_{2}-x_{1}, \ldots, x_{j}-x_{1}\right\rangle R_{s},
\end{gathered}
$$

$h$ the image of $f_{1}$ in $B[T]$; and again by (6), $h$ is a prime element.

To establish (c) it will be arranged that for every permutation $\pi \in S_{n}$, $\left\langle f_{\pi(1)}, \ldots, f_{\pi(j)}, f\right\rangle$ and $\left\langle f_{\pi(1)}, \ldots, f_{\pi(j)}\right\rangle$ are prime ideals $(1 \leqslant j \leqslant n)$. Further restrict $s$ so that for each such $\pi, s$ satisfies, relative to the sequence $\left(x_{\pi(1)}, \ldots, x_{\pi(n)}\right)$, the conditions imposed above relative to the sequence $\left(x_{1}, \ldots, x_{n}\right)$. Now the sequence $\left(f_{\pi(1)}, \ldots, f_{\pi(n)}\right)$ is exactly the one assigned by the above rule to the sequence $\left(x_{\pi(1)}, \ldots, x_{\pi(n)}\right)$. The desired result now follows from Claim (iii). Q.E.D. for (c), and hence for (1).

(1) says that when $A_{M}$ is regular, $M$ has a minimal basis with the properties described in (2). Question: Does $M$ have such a basis even if $A_{M}$ is not regular? Perhaps so, but the above methods could not possibly prove it. These methods would at least require the existence of a minimal basis for $P R_{P}$ 
containing "enough" prime sequences; but unfortunately $P R_{P}$ may contain no prime elements. But $P R_{P}$ will have a good enough basis if $R_{P}$ is the localization of a geometric domain of the type considered in (2). Hence there exists the following corollary of the proof of (1) and the omitted proof of (2). The proof of the corollary is also omitted.

(7) CoRollary. Let $A=R[T], R$ an affine algebra over an algebraically closed field, and let $M$ be a maximal ideal of $A$ such that $A_{M}$ is a domain. Then $M$ has a minimal basis with the property that each of its nonempty subsets having cardinality less than $\operatorname{grade}(M)$ generates a prime ideal.

ADDED IN PROOF (May 1978). The prime sequence question has been settled in the negative by $R$. Heitmann (personal communication). That the prime sequence question has an affirmative answer in affine domains over infinite fields also follows from the Ph.D. dissertation of N. V. Trung (Halle, 1977).

\section{REFERENCES}

[AT] E. Artin and J. Tate, A note on finite ring extensions, J. Math. Soc. Japan 3 (1951), 74-77.

[BDG] M. Boratyński, E. D. Davis and A. V. Geramita, Generators for certain ideals in regular polynomials rings of dimension three, J. Algebra 51 (1978), 320-325.

[DG] E. D. Davis and A. V. Geramita, Efficient generation of maximal ideals in polynomial rings, Trans. Amer. Math. Soc. 231 (1977), 497-505.

[E] S. Endo, Projective modules over polynomial rings, J. Math. Soc. Japan 15 (1963), 339-352.

[K] I. Kaplansky, Commutative rings, Allyn and Bacon, Boston, Mass., 1970.

[Sg] A. Seidenberg, The hyperplane sections of normal varieties, Trans. Amer. Math. Soc. 69 (1950), 357-386.

[Sn] R. G. Swan, A cancellation theorem for projective modules in the metastable range, Invent. Math. 27 (1974), 23-43.

[Z] O. Zariski, Foundations of a general theory of birational correspondence, Trans. Amer. Math. Soc. 53 (1943), 490-542.

Department of Mathematics, State University of New York at Albany, Albany, New YORK 12222 